\title{
Bright Solitons in Asymmetrically Trapped Bose-Einstein Condensates
}

\author{
Sk. Golam Ali, B. Talukdar* And S.K. Roy \\ Department of Physics, Visva-Bharati University, Santiniketan 731235, India
}

(Received February 12, 2007)

\begin{abstract}
We study the dynamics of bright solitons in a Bose-Einstein condensate confined in a highly asymmetric trap. While working within the framework of a variational approach we carry out the stability analysis of the BoseEinstein condensate solitons against collapse. When the number of atoms in the soliton exceeds a critical number $N_{\mathrm{c}}$, it undergoes the so-called primary collapse. We find an analytical expression for $N_{\mathrm{c}}$ in terms of appropriate experimental quantities that are used to produce and confine the condensate. We further demonstrate that, in the geometry of the problem considered, the width of the soliton varies inversely as the number of constituent atoms.
\end{abstract}

PACS numbers: 03.75.-b, 03.75.Kk, 05.30.Jp

\section{Introduction}

A Bose-Einstein condensate (BEC) consists of trapped ultracold atoms all in the same quantum state. In this state the atoms lose their individual identities and behave as a single collective wave which is large enough to be optically imaged. In order to create a BEC, atoms are first confined within a strong magnetic field and then the temperature of the atomic gas is continually lowered by laser and evaporative cooling until the condensate is formed. If one confines the BEC in only two directions, it will tend to disperse in the free direction. Because of the energetics involved, the atom-atom interaction in freely propagating BEC is characterized by the $s$-wave scattering length. The Feshbach resonance [1] allows one to continuously tune the scattering length from a positive to negative value (repulsive to attractive interaction) by means of applied magnetic field. For attractive atomic interaction we can have coherently propagating matter-wave packets which travel over the BEC with neither attenuation nor change in shape. These are the so-called bright solitons. For repulsive interaction we shall have dark solitons. Understandably, a bright soliton is a peak on the BEC while a dark soliton is

*corresponding author; e-mail: binoy123@sancharnet.in 
a notch with a characteristic phase step across it. In a BEC of ${ }^{7} \mathrm{Li}$ atom Rice and Paris teams [2] produced bright solitons, each of which represents a condensate of actual atoms extracted from the main BEC.

When the number of atoms in a bright soliton exceeds a critical value, it becomes unstable due to focusing nonlinearity arising from the attractive atomatom interaction. The transverse dimensions of the confinement then cause the soliton to collapse. This is often referred to as the primary collapse [3]. In the present work we shall envisage a variational study for the stability of bright soliton in a highly elongated trap and thereby calculate the critical number of atoms $\left(N_{\mathrm{c}}\right)$ that a soliton can hold before it undergoes the so-called primary collapse. We shall see that the merit of our approach is its directness and simplicity because the variational method sought by us provides a straightforward analytical model to understand the dynamics of bright solitons.

To extract the relevant physical information regarding stability and/or collapse we shall work within the framework of a mean field approximation. In this approximation the dynamics of a BEC is modelled by 3D Gross-Pitaevskii (GP) equation

$$
\mathrm{i} \hbar \frac{\partial \psi}{\partial t}=-\frac{\hbar^{2}}{2 m} \nabla^{2} \psi+V(\boldsymbol{r}) \psi+U_{0}|\psi|^{2} \psi,
$$

where $\psi(\boldsymbol{r}, t)$ is the macroscopic wave function of the condensate. This wave function is also called the order parameter. Here $U_{0}=4 \pi \hbar^{2} a_{\mathrm{s}} / m$ represents the interatomic interaction with $a_{\mathrm{s}}$, the two-particle $s$-wave scattering length and $m$, the mass of the atom. The wave function $\psi$ is normalized to the number of particles $N$ in the condensates such that

$$
\int|\psi|^{2} \mathrm{~d} \boldsymbol{r}=N
$$

The potential $V(\boldsymbol{r})$ confines the atoms in a trap. For harmonic trapping, $V(\boldsymbol{r})$ is given by

$$
V(\boldsymbol{r})=\frac{1}{2} m \nu^{2}\left(\lambda_{x}^{2} x^{2}+\lambda_{y}^{2} y^{2}+\lambda_{z}^{2} z^{2}\right) .
$$

The parameters $\lambda_{x}, \lambda_{y}$, and $\lambda_{z}$ describe anisotropy of the trap in the $x, y$, and $z$ direction, respectively. We shall work with a highly asymmetric trap as determined by $\lambda_{x}=\lambda_{y}=1$ and $\lambda_{z}=\nu_{z} / \nu \ll 1$. Here $\nu_{z}$ represents the frequency along the $z$ directions and $\nu=\nu_{\mathrm{r}}$, the radial frequency. Our system of interest is thus a quasi-one-dimensional (Q1D) BEC dispersing along the $z$ direction. In the following we derive an appropriate version of the GP equation that will be useful to study the dynamics of BEC in highly asymmetric traps.

We consider (1) in a geometry in which the trapping potential in $z$ is much weaker than the corresponding potential in $r=\left(x^{2}+y^{2}\right)^{\frac{1}{2}}$. Further, we write the equation in terms of dimensionless variables defined by 


$$
\tau=\nu t, \quad \rho=\frac{r}{a_{0}}, \quad s=\frac{z}{a_{0}}, \quad \psi(r, z, t)=\frac{u(\rho, s, \tau)}{a_{0}^{\frac{3}{2}}} .
$$

This gives

$$
\mathrm{i} u_{\tau}+\frac{1}{2} \nabla^{2} u-\frac{1}{2}\left(\rho^{2}+\lambda_{z}^{2} s^{2}\right) u-\frac{4 \pi a_{\mathrm{s}}}{a_{0}}|u|^{2} u=0 .
$$

Here $a_{0}=\sqrt{\hbar / m \nu}$ is the size of the ground state solution of the noninteracting GP equation. It is obvious that

$$
\int|u|^{2} \mathrm{~d}^{3} \rho=N
$$

We assume a separable ansatz for the solution of (5) such that [4]

$$
u(\rho, s, \tau)=\phi(\rho) \xi(s, \tau) .
$$

From (5) and (7) we have

$$
\frac{1}{\xi}\left(\mathrm{i} \xi_{\tau}+\frac{1}{2} \xi_{2 s}-\frac{1}{2} \lambda_{z}^{2} s^{2} \xi\right)-\frac{4 \pi a_{\mathrm{s}}}{a_{0}}|\xi|^{2}|\phi|^{2}=\frac{1}{\phi}\left(-\frac{1}{2} \nabla_{\rho}^{2} \phi+\frac{1}{2} \rho^{2} \phi\right),
$$

where $\nabla_{\rho}^{2}$ stands for the Laplacian in the radial coordinate. In (8) the subscripts on $\xi$ stand for partial derivative with respect to that particular independent variable. More specifically, $\xi_{2 s}=\partial^{2} \xi / \partial s^{2}$. This equation shows that the presence of atom-atom interaction does not permit clearcut separation of variables. However, the fourth term in Eq. (8) is quite small. Thus, $\phi$ may be assumed to satisfy

$$
-\frac{1}{2} \nabla_{\rho}^{2} \phi+\frac{1}{2} \rho^{2} \phi=\nu_{\rho} \phi
$$

with $\nu_{\rho}$ being related to $\nu_{\mathrm{r}}$ by a scale factor determined by the change of variables sought in (4). Equation (9) represents the well-known eigenvalue problem for the two-dimensional harmonic oscillator with the ground state solution given by

$$
\phi_{0}(\rho)=\mathrm{e}^{-\rho^{2} / 2} \text {. }
$$

Combining (8) and (9) we write

$$
\mathrm{i} \xi_{\tau}+\frac{1}{2} \xi_{2 s}-\frac{1}{2} \lambda_{z}^{2} s^{2} \xi-\frac{4 \pi a_{\mathrm{s}}}{a_{0}}|\xi|^{2}|\phi|^{2} \xi=\nu_{\rho} \xi .
$$

The low-frequency vibration along the $z$ direction is quite unlikely to excite the two-dimensional bosonic oscillator from its ground state. Thus (11) can be multiplied by $\phi \phi^{*}$ and integrated over the $\rho$ coordinate to get

$$
\mathrm{i} \xi_{\tau}+\frac{1}{2} \xi_{2 s}-\frac{1}{2} \lambda_{z}^{2} s^{2} \xi-\frac{2 \pi a_{\mathrm{s}}}{a_{0}}|\xi|^{2} \xi=\nu_{\rho} \xi
$$

Equation (12) represents the GP equation for a Q1D trap. For a true 1D system one does not expect the collapse of the system with increasing number of atoms. But the use of Q1D trap in controlling the condensate motion may result in the collapse of a BEC soliton when the number of atoms in it exceeds a critical value, say, $N_{\mathrm{c}}$. We shall work with (12) to provide an analytical model to study the collapse dynamics of bright solitons in a Q1D trap. Interestingly, Eq. (12) can be written in a more convenient form by using the change of variable 


$$
\xi(s, \tau)=\chi(s, \tau) \exp \left(-\mathrm{i} \nu_{\rho} \tau\right)
$$

From (12) and (13) we get

$$
\mathrm{i} \chi_{\tau}+\frac{1}{2} \chi_{2 s}-\frac{1}{2} \lambda_{z}^{2} s^{2} \chi-\frac{2 \pi a_{\mathrm{s}}}{a_{0}}|\chi|^{2} \chi=0
$$

with

$$
\int_{-\infty}^{+\infty}|\chi|^{2} \mathrm{~d} s=N / \pi
$$

Equation (14) represents a desired form of the evolution equation in which the atom-atom interaction is characterized by a negative scattering length. The realistic 1D limit in (14) is not a true 1D system because this equation involves the effect of transverse degrees of freedom through $\lambda_{z}$ and $a_{0}$. In this context we note that a similar equation with $\psi(r, z, t)$ chosen as $u(\rho, s, \tau) / \sqrt{a_{0}^{3} / N}$ was used by Pérez-García et al. [4] to qualitatively demonstrate that if the number of particles is large enough, the condensate is unstable and the collapse occurs. We are, however, interested to derive a straightforward analytical model to understand the collapse dynamics and thereby provide a quantitative estimate for $N_{\mathrm{c}}$. To that end we convert, in Sect. 2, the initial-boundary value problem in (14) to a variational problem. In particular, we present an expression for the Lagrangian density and a trial wave function involving variational parameters to study the stability of bright solitons against collapse. We also obtain the evolution equations for these parameters. In Sect. 3 we judiciously use the derived evolution laws to study the soliton dynamics with particular emphasis on the stability of solitons against collapse.

\section{Variational formulation}

The action principle

$$
\delta \iint \mathcal{L}\left(\chi, \chi^{*}, \chi_{s}, \chi_{s}^{*}, \chi_{\tau}, \chi_{\tau}^{*}\right) \mathrm{d} s \mathrm{~d} \tau
$$

with the Lagrangian density given by

$$
\mathcal{L}=\frac{\mathrm{i}}{2}\left(\chi \chi_{\tau}^{*}-\chi^{*} \chi_{\tau}\right)+\frac{1}{2} \lambda_{z}^{2} s^{2} \chi \chi^{*}+\frac{\pi a_{\mathrm{s}}}{a_{0}} \chi^{2} \chi^{* 2}+\frac{1}{2} \chi_{s}^{*} \chi_{s}
$$

is equivalent to (14). We shall use this expression for $\mathcal{L}$ to study the dynamics of bright solitons in terms of a variational method often called the Ritz optimization procedure [5]. In this procedure the first variation of the variational functional is made to vanish within a set of suitable chosen trial functions such that the field theoretical problem under consideration reduces to a simple problem of point mechanics. For the negative scattering length the Gaussian trial function for $\chi(s, \tau)$ is a very reasonable ansatz. Thus we write

$$
\chi(s, \tau)=A(\tau) \exp \left(-s^{2} / 2 a^{2}(\tau)+\mathrm{i} b(\tau) s^{2} / 2\right) .
$$

Here $A(\tau)$ is a complex amplitude, $a(\tau)$ - the width of the distribution and $b(\tau)$ - the frequency chirp. The phase of the condensate $\delta(\tau)$ is defined by $A(\tau)=$ $|A(\tau)| \mathrm{e}^{\delta(\tau)}$. The amplitude $A(\tau)$, width $a(\tau)$, and the chirp $b(\tau)$ will all vary with the time parameter $\tau$. The initial condensate at rest will have $\mathrm{d} a(\tau) / \mathrm{d} \tau=0$. 
Inserting the trial function in (18) into the variational principle stated in (16) we obtain a reduced variational problem

$$
\delta \int\langle\mathcal{L}\rangle \mathrm{d} \tau=0
$$

with

$$
\langle\mathcal{L}\rangle=\int_{-\infty}^{+\infty} \mathcal{L}_{\mathrm{G}} \mathrm{d} s .
$$

Here $\mathcal{L}_{\mathrm{G}}$ represents the result obtained by inserting the Gaussian ansatz (18) into the Lagrangian density in (17). It is rather straightforward to perform the integration in (20) and get

$$
\begin{aligned}
& \langle\mathcal{L}\rangle=\sqrt{\pi}\left[\frac{i}{2}\left(A A_{\tau}^{*}-A^{*} A_{\tau}\right) a+\frac{1}{4} b_{\tau} a^{3} A A^{*}\right. \\
& \left.+\frac{1}{4} \lambda_{z}^{2} a^{3} A A^{*}+\frac{\pi}{\sqrt{2}} \frac{a_{\mathrm{s}}}{a_{0}} a A^{2} A^{* 2}+\frac{1}{4}\left(\frac{1}{a}+b^{2} a^{3}\right) A A^{*}\right] .
\end{aligned}
$$

Let us now obtain the variational equations for the Gaussian parameters $A(\tau), A^{*}(\tau), a(\tau)$, and $b(\tau)$ which follow from the vanishing conditions of $\delta\langle\mathcal{L}\rangle / \delta A$, $\delta\langle\mathcal{L}\rangle / \delta A^{*}, \delta\langle\mathcal{L}\rangle / \delta a$, and $\delta\langle\mathcal{L}\rangle / \delta b$. These equations are given by

$$
\begin{aligned}
\frac{\delta\langle\mathcal{L}\rangle}{\delta A} & =\mathrm{i} A_{\tau}^{*} a+\frac{\mathrm{i}}{2} A^{*} a_{\tau}+\frac{1}{4} b_{\tau} a^{3} A^{*}+\frac{1}{4} \lambda_{z}^{2} a^{3} A^{*} \\
& +\pi \sqrt{2} \frac{a_{\mathrm{s}}}{a_{0}} a A A^{* 2}+\frac{1}{4}\left(\frac{1}{a}+b^{2} a^{3}\right) A^{*}=0, \\
\frac{\delta\langle\mathcal{L}\rangle}{\delta A^{*}} & =-\mathrm{i} A_{\tau} a-\frac{\mathrm{i}}{2} A a_{\tau}+\frac{1}{4} b_{\tau} a^{3} A+\frac{1}{4} \lambda_{z}^{2} a^{3} A \\
& +\pi \sqrt{2} \frac{a_{\mathrm{s}}}{a_{0}} a A^{2} A^{*}+\frac{1}{4}\left(\frac{1}{a}+b^{2} a^{3}\right) A=0, \\
\frac{\delta\langle\mathcal{L}\rangle}{\delta a} & =\frac{\mathrm{i}}{2}\left(A A_{\tau}^{*}-A^{*} A_{\tau}\right)+\frac{3}{4} b_{\tau} a^{2} A A^{*} \\
& +\frac{3}{4} \lambda_{z}^{2} a^{2} A A^{*}+\frac{\pi}{\sqrt{2}} \frac{a_{\mathrm{s}}}{a_{0}} A^{2} A^{* 2}+\frac{1}{4}\left(-\frac{1}{a^{2}}+3 b^{2} a^{2}\right) A A^{*}=0
\end{aligned}
$$

and

$$
\frac{\delta\langle\mathcal{L}\rangle}{\delta b}=\frac{1}{2} b a^{3} A A^{*}-\frac{1}{4} \frac{\partial}{\partial \tau}\left(a^{3} A A^{*}\right)=0 .
$$

From (22) and (23) we have found

$$
\frac{\mathrm{d}}{d \tau}\left(a A A^{*}\right)=0
$$

such that

$$
a|A|^{2}=Q, \text { a constant. }
$$

The constant $Q$ is simply related to the number of particles in the condensate 
since the value of the integral (15) is $\sqrt{\pi} a|A|^{2}$. Combining (25) and (27) we get

$$
b=\frac{\mathrm{d}}{d \tau}(\ln a) .
$$

Equations (27) and (28) clearly show that if we can derive a method to calculate the values of $a(\tau)$, the other parameters of the condensate will be automatically determined. Fortunately, (22), (23), (24), and (28) can be combined to write a second-order ordinary differential equation, the first integral of which gives

$$
\frac{1}{2}\left(\frac{\mathrm{d} a}{\mathrm{~d} \tau}\right)^{2}+\frac{1}{2} \lambda_{z}^{2} a^{2}+\sqrt{\frac{2}{\pi}} \frac{N a_{\mathrm{s}}}{a_{0}} \frac{1}{a}+\frac{1}{2 a^{2}}=E,
$$

with $E$, the constant of integration.

The equation for $a(\tau)$ in $(29)$ is related to the motion of a particle in a potential field $V(a)$ so that

$$
\frac{1}{2}\left(\frac{\mathrm{d} a}{\mathrm{~d} \tau}\right)^{2}+V(a)=E .
$$

Here

$$
V(a)=\frac{1}{2} \lambda_{z}^{2} a^{2}+\frac{P}{a}+\frac{1}{2 a^{2}}, \quad P=\sqrt{\frac{2}{\pi}} \frac{N a_{\mathrm{s}}}{a_{0}} .
$$

Thus one would like to interpret the constant of the motion $E$ as the total energy of the particle. It is easy to verify that there is no physical uncertainty in the identification sought because $\int_{-\infty}^{+\infty} \mathcal{H}_{\mathrm{G}} \mathrm{d} s$ represents the left side of $(29)$. Here $\mathcal{H}_{\mathrm{G}}$ stands for the result of the Hamiltonian density calculated from (18) and rewritten by using the Gaussian ansatz. Obviously, $E$ is determined by the initial conditions of the second-order differential equation from which (30) has been extracted. It is not difficult to solve (30) and look for the dynamics of the condensate. However, the analysis of the equilibrium point obtained from the extremum of $V(a)$ written as

$$
\frac{\mathrm{d} V(a)}{\mathrm{d} a}=0
$$

can give some illuminating results.

\section{Dynamics of bright solitons}

For bright solitons the nonlinear interaction is attractive and the scattering length $a_{\mathrm{s}}<0$. In this case we shall use $P=-|P|$ and carry out the subsequent analysis by using only the numerical values of $a_{\mathrm{s}}$. We shall make use of (32) to derive a simple physical picture for the collapse dynamics of bright solitons when the trap of the BEC is relaxed in one direction. From (31) and (32) with $P=-|P|$ we get

$$
\lambda_{z}^{2} a^{4}+|P| a-1=0 .
$$

The equilibrium point determined by (33) should be a minimum for (14) to support a soliton solution. This gives

$$
\lambda_{z}^{2} a^{4}-2|P| a+3=\beta a^{4}, \quad \beta>0 .
$$


Eliminating $|P|$ from (33) and (34) we find that

$$
a=\frac{1}{\left(\beta-3 \lambda_{z}^{2}\right)^{\frac{1}{4}}}, \quad \beta>3 \lambda_{z}^{2},
$$

is a particular solution of (33) and (34). From (35) and (33) or (34) we get

$$
|P|=\frac{\beta-4 \lambda_{z}^{2}}{\left(\beta-3 \lambda_{z}^{2}\right)^{\frac{3}{4}}} .
$$

The form of (36) imposes a further restriction on the values of $\beta$ than that given in (35) and sets a lower bound for it. Using $\beta=\gamma \lambda_{z}^{2}$ we write (36) in the form

$$
|P|=\frac{\gamma-4}{(\gamma-3)^{\frac{3}{4}}} \sqrt{\lambda_{z}}
$$

Thus non-zero values of $P$ will be obtained for $\gamma>4$ only. For $\gamma=4$ the interaction term vanishes and GP equation becomes linear and the soliton formation becomes impossible.

From (31) and (37) we obtain an expression

$$
N=\sqrt{\frac{\pi}{2}} \frac{a_{0}}{\left|a_{\mathrm{s}}\right|} \frac{\gamma-4}{(\gamma-3)^{\frac{3}{4}}} \sqrt{\lambda_{z}}
$$

for the number of atoms in the Q1D soliton.

In Fig. 1 we plot the potential $V(a)$ in (31) as a function of $a$ for $a_{\mathrm{s}}=$ $-1.59 \times 10^{-4} \mu \mathrm{m}$, the scattering length of ${ }^{7} \mathrm{Li}$ as used in the experiment of Strecker et al. [6]. We have chosen to work with $\lambda_{z}=\frac{4}{400}$. In this figure we have four curves represented by $V_{4}(a), V_{5}(a), V_{6}(a)$, and $V_{7}(a)$ corresponding to $\gamma=4,5,6$, and 7 , respectively. A common feature of all these potentials is that each of them exhibits a minimum. The curve for $V_{7}(a)$ represents a potential well between $a_{1}=5.0114$ and $a_{2}=10.5468$. The minimum of the well is negative. A mechanical analogy suggests a solution which oscillates between the zeros of $V_{7}(a)$. In this case, the spreading of the BEC is stopped at $a=a_{2}$ by nonlinear effects which subsequently compress the BEC back to the initial width. This behavior is repeated in an oscillatory manner. In this situation the BEC soliton will become unstable and lead to a mechanical collapse [7]. A similar situation arises for other values of $\gamma>6$.

For $\gamma=6$ the potential well degenerates into a single point such that $V_{6}(a)$ touches the $a$ axis at a particular point, where the potential has a stable minimum. Understandably, a particle released at this point will stay there. In the present context this implies that for our chosen value of $\lambda_{z}$ and $\gamma=6$ the BEC bright soliton will be critically stable. Using $\gamma=6$ we get

$$
N_{\mathrm{c}}=0.8774 \sqrt{\frac{\pi}{2}} \frac{a_{0}}{\left|a_{\mathrm{s}}\right|} \sqrt{\lambda_{z}} .
$$

After the number of atoms exceeds this critical number, the soliton becomes unstable. More than a decade ago Ruprecht et al. [8] used a purely numerical routine to set a limit for the critical number of atoms after which the BEC with the attractive 


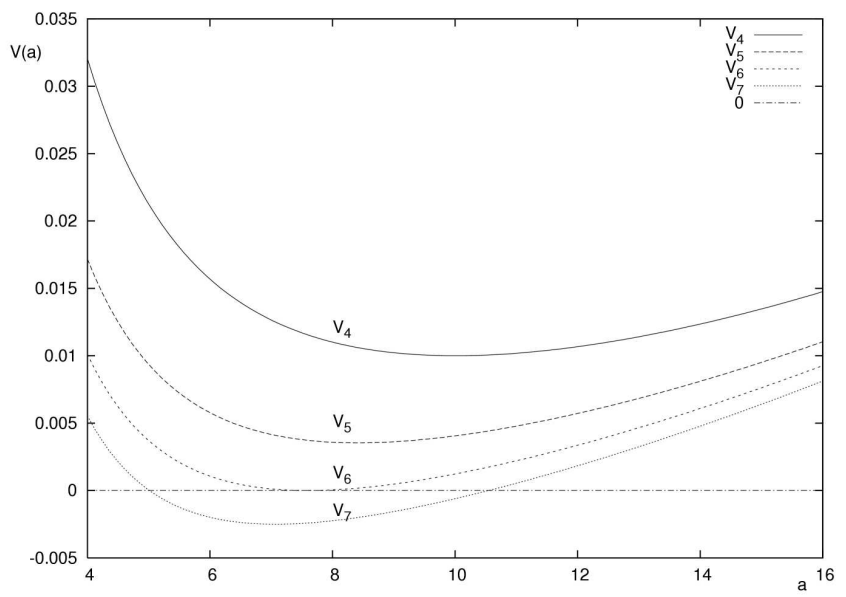

Fig. 1. The potential $V(a)$ as a function of $a$ for $\lambda_{z}=\frac{1}{100}$.

two-body interaction becomes unstable. This limit was examined by Gammal et al. [9] for different trapping geometries. Interestingly, the analytical expression in (39) is in agreement with the observations of Refs. [8] and [9]. From (39) it is clear that $N_{\mathrm{c}}\left|a_{\mathrm{s}}\right| / a_{0} \ll 1$. This represents the well-known relation for the existence of stable solitons [10].

It will be interesting to see what happens if $\gamma<6$. In order to see that we look at the curve represented by $V_{4}(a)$. For $\gamma=4,|P|=0$ we do not have a nonlinear term in the GP equation. In this case, no soliton can be formed. The minimum of $V_{4}$ is positive. This appears to suggest that weaker nonlinearities leading to potential curves with positive minima will not be able to produce matter-wave bright solitons. In our figure $V_{5}(a)$ represents one such curve.

Equations (35) and (37) can be combined to write

$$
a(\tau)=\frac{C}{N}
$$

where

$$
C=\sqrt{\frac{\pi}{2}} \frac{\gamma-4}{\gamma-3} \frac{a_{0}}{\left|a_{\mathrm{s}}\right|}
$$

The result in (40) is remarkable and implies that, at a given instant of time, if the number of atoms in the soliton increases, it becomes narrower. It is a real curiosity to note that the result in (40) holds good even in the absence of trapping [4].

\section{Acknowledgments}

One of the authors (S.G.A.) is thankful to the UGC, Govt. of India for a Research Fellowship. 


\section{References}

[1] J.L. Roberts, N.R. Claussen, James P. Burke, Jr., Chris H. Greene, E.A. Cornell, C.E. Wieman, Phys. Rev. Lett. 81, 5109 (1998); D.E. Pelinovsky, P.G. Kevrekidis, D.J. Frantzeskakis, Phys. Rev. Lett. 91, 240201 (2004).

[2] K.E. Strecker, G.B. Partridge, A.G. Truscott, G.B. Hulet, Nature (London) 417, 150 (2002); L. Khaykowich, F. Schreck, G. Ferrari, T. Bourdel, J. Cubizoller, L.D. Carr, Y. Castin, C. Salomon, Science 296, 1290 (2002).

[3] L.D. Carr, J. Brand, Phys. Rev. Lett. 92, 040401 (2004).

[4] V.M. Pérez-García, H. Michinel, H. Herrero, Phys. Rev. A 57, 3837 (1998).

[5] G.B. Arfken, H.J. Weber, Mathematical Method for Physicists, Elsevier, New Delhi 2004.

[6] L. Salasnich, A. Parola, L. Reatto, Phys. Rev. A 66, 043603 (2002); K.E. Strecker, G.B. Partridge, A.G. Truscott, G.B. Hulet, New. J. Phys. 5, 73.1 (2003).

[7] F. Dalfovo, S. Giorgini, L.P. Pitaevskii, S. Stringari, Rev. Mod. Phys. 71, 463 (1999).

[8] A.P. Ruprecht, M.J. Holland, K. Burnett, M. Edwards, Phys. Rev. A 51, 4704 (1995).

[9] A. Gammal, T. Frederico, L. Tomio, Phys. Rev. A 64, 055602 (2001).

[10] F. Kh. Abdullaev, A. Gammal, A.M. Kamchatnov, L. Tomio, Int. J. Mod. Phys. $B$ 19, 3415 (2005). 